\title{
Epidemiology of kidney stone and bacterial strains with antibiotic resistance in Shiraz, southwest of Iran, 2014-2019
}

\author{
Farshad Kakian ${ }^{1}$, Mohammad Ghasemi Palangi ${ }^{2}$, Nahal Hadi ${ }^{1, *}$ \\ ${ }^{1}$ Department of Bacteriology and Virology, School of Medicine, Shiraz University of Medical \\ Sciences, Shiraz, Iran \\ ${ }^{2}$ School of Medicine, Shiraz University of Medical Sciences, Shiraz, Iran
}

*. Corresponding author: Nahal Hadi, Department of Bacteriology and Virology, School of Medicine, Shiraz University of Medical Sciences, Shiraz, Iran. E-mail: hadina@sums.ac.ir.

Cite this article: Kakian, F.; Ghasemi Palangi, M.; Hadi, N. kidney stone and bacterial strains with antibiotic resistance in Shiraz, southwest of Iran, 2014-2019. Int J Epidemiol Health Sci 2021;2(3): e08. Doi:10.51757/IJEHS.2.3.2021.47993

\begin{abstract}
Background: Urinary stones are one of the major health problems worldwide and a significant part of referrals to physicians. Also, growing antibiotics resistance is an important concern. The purpose of this study was to evaluate the prevalence of urinary stones and bacterial strains with antibiotic resistance in women admitted to shiraz, southwest of Iran, from 2014 to 2019.

Methods: In this period, all women admitted to the Zeinabieh Hospital were evaluated. The frequency of urinary stones and bacterial strains and antibiogram patterns of bacterial isolates were investigated from 2014 to 2019. Antibacterial susceptibility testing was done by disk diffusion method, fulfilling the standard criteria of "Clinical and Laboratory Standards Institute (CLSI) Performance Standards for Antimicrobial Susceptibility".

Results: Out of 101 isolates, the identified gram-negative bacteria were E. coli (68.3\%), Klebsiella (6.9\%), Enterobacter (6.9\%), Pseudomonas (5\%), Proteus (1\%), and Acinetobacter (1\%). Of gram-positive strains, Coagulase-positive Staphylococci (5.9\%), coagulase-negative Staphylococcus (CONS) (3\%) and Non-Hemolytic Streptococci $(2 \%)$ were identified. Also, the frequency of urinary tract stones was about $5 \%$. Four cases of kidney stones were associated with E. coli and one case with Klebsiella. All isolates had the highest resistance to cotrimoxazole (62.5\%), Ampicillin and Nalidixic acid.

Conclusion: In this study, no association was found between the type of bacteria and urinary stone. From the variable bacteria isolated, the highest rate of $E$. coli (as an important pathogen) was confirmed. Also, most of the bacterial isolates were resistant to multiple antibiotics, although Amikacin and nitrofurantoin (as the empirical therapy in our hospital) were dominated.
\end{abstract}

Keywords: Urinary (kidney) stone, Epidemiology, Bacterial strains, Antimicrobial resistance, Iran

\section{Introduction}

Bacterial infections pose a serious threat to the health of society and individuals. Urinary tract infections are one of these infections (1). Most women experience this condition at least once in their lifetime. The incidence of urinary tract infections in adult women is about 30 times higher than men $(2,3)$.

Kidney stone disease, too recognized as nephrolithiasis or urolithiasis, is when a solid piece of material (kidney stone) grows in the urinary tract (4). Urinary stones are the third most common affliction of the urinary tract. These stones can increase in urinary tract infections and pathological conditions of the prostate (5).The occurrence of kidney stone has increased during the 20th century. It happens in up to $15 \%$ of the population of the United States. The rate of new cases and recurrences may continue to increase. So, new methods in treatment and prevention could 
have a vast economic influence over in terms of reduced morbidity (6).

Among the bacteria that cause urinary tract infections; Urease-producing species such as Proteus, Klebsiella, and Pseudomonas cause most infectious stones. Also, microorganisms that do not produce urease, such as Escherichia coli and Enterobacter, with different mechanisms can be involved in the formation of urethral stones (7-9). In the absence of control and increasing antibiotic resistance, we will face an increase in antibiotic-resistant bacterial species and an increase in treatment failure $(10,11)$.

Urinary stones have been considered as one of the health problems in our country and are a significant part of referrals to doctors. Urinary tract infections, which are common in women, are also a cause of economic and medical problems. The purpose of this study was to evaluate the prevalence of urinary stones and bacterial strains with antibiotic resistance in women admitted to shiraz from 2014 to 2019.

\section{Methods}

This retrospective study was done on records of the cultures and ultrasound done for women admitted to the Zainabieh Hospital, a chief teaching hospital affiliated to Shiraz University of Medical Sciences, and the main referral center of women in southwest of Iran during the period of 2014-2019.

A checklist was designed to collect data including age, culture, antibiotic sensitivity test and ultrasound of the urinary system results. Culturing done on blood agar and MacConkey agar. Bacteria that have grown from culture were recognized by morphology features, gram staining and biochemical tests such as Triple Sugar Iron test (TSI), Simon citrate, Methyl Red / VogesProskauer (MR/VP), SIM (Sulfide, Indole, Motility), Oxidase and Catalase, etc.

\section{Percent}

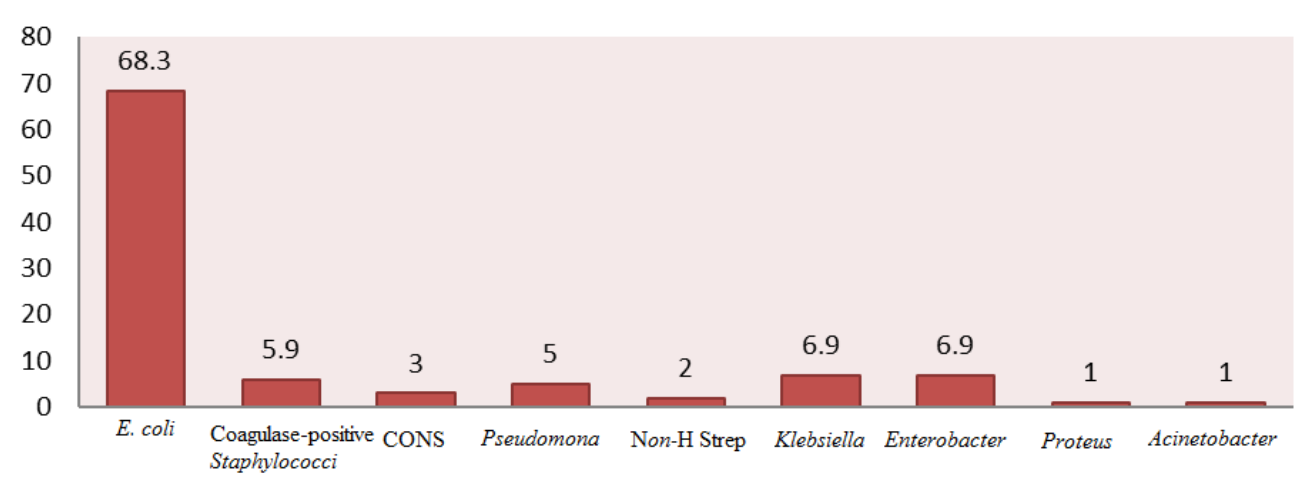

Bacteria
The disk diffusion method (Kirby-Bauer) was used to determine the antimicrobial susceptibility on MuellerHinton agar medium according to guidelines of Clinical Laboratory Standards Institute (CLSI).

The performed antibiogram disks included Ciprofloxacin (CIP, $5 \mathrm{mcg}$ ), Gentamicin (GM, 10 $\mathrm{mcg}$ ), Imipenem (IPM, 10mcg), Vancomycin (V, 30 $\mathrm{mcg}$ ), Amikacin (AMK, $30 \mathrm{mcg}$ ), Cefixime (CFM), Cotrimoxazole (CTX $25 \mathrm{mg}$ ), Cefepime (FEP), Nalidixic acid (NAL), Ampicillin (AMP) and Nitrofurantoin (NF).

For analyses data used a SPSS 21.0 software. Also, Student's t-test and pair t test used for data. The variables were expressed by number and percent and Chi-square test was carried out to highlighting the associations between them. In all tests the significance level and relation between variables was defined as $\mathrm{P}<0.05$.

\section{Results}

In this study, by reviewing the records of all patients, 101 cases of UTI were observed. The mean age of patients was 36.58, with a standard deviation of 16.72 years (Minimum: 18 years; Maximum: 91 years). The median age of patients was 31 years.

\section{Bacteria isolated}

In our study, Escherichia coli was the most common bacterium with $68.3 \%$, followed by Klebsiella spp. and Enterobacter with 6.9\%. Also, among grampositives bacteria, coagulase-positive Staphylococcus was the most frequency (figure 1). In all years, E. coli has been the most common bacterium with $60 \%$. In addition, no significant difference was found between the year and the type of bacteria.

Figure 1. Distribution of bacteria strains from 2014 to 2019 CONS: Coagulase-negative Staphylococcus 


\section{Percent}

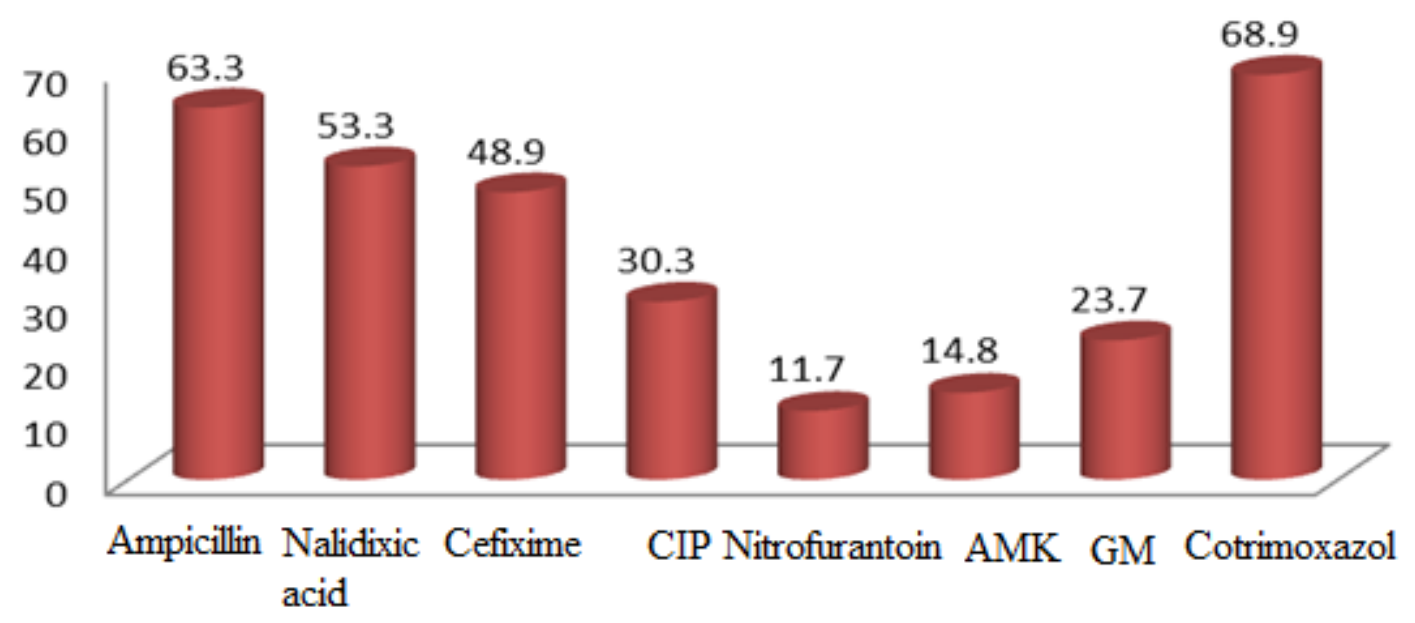

\section{Antibiotic}

Figure 2. Antibiotic resistance in E. coli

$\mathrm{CIP}=$ Ciprofloxacin, $\mathrm{AMK}=$ Amikacin, and $\mathrm{GM}=$ Gentamicin

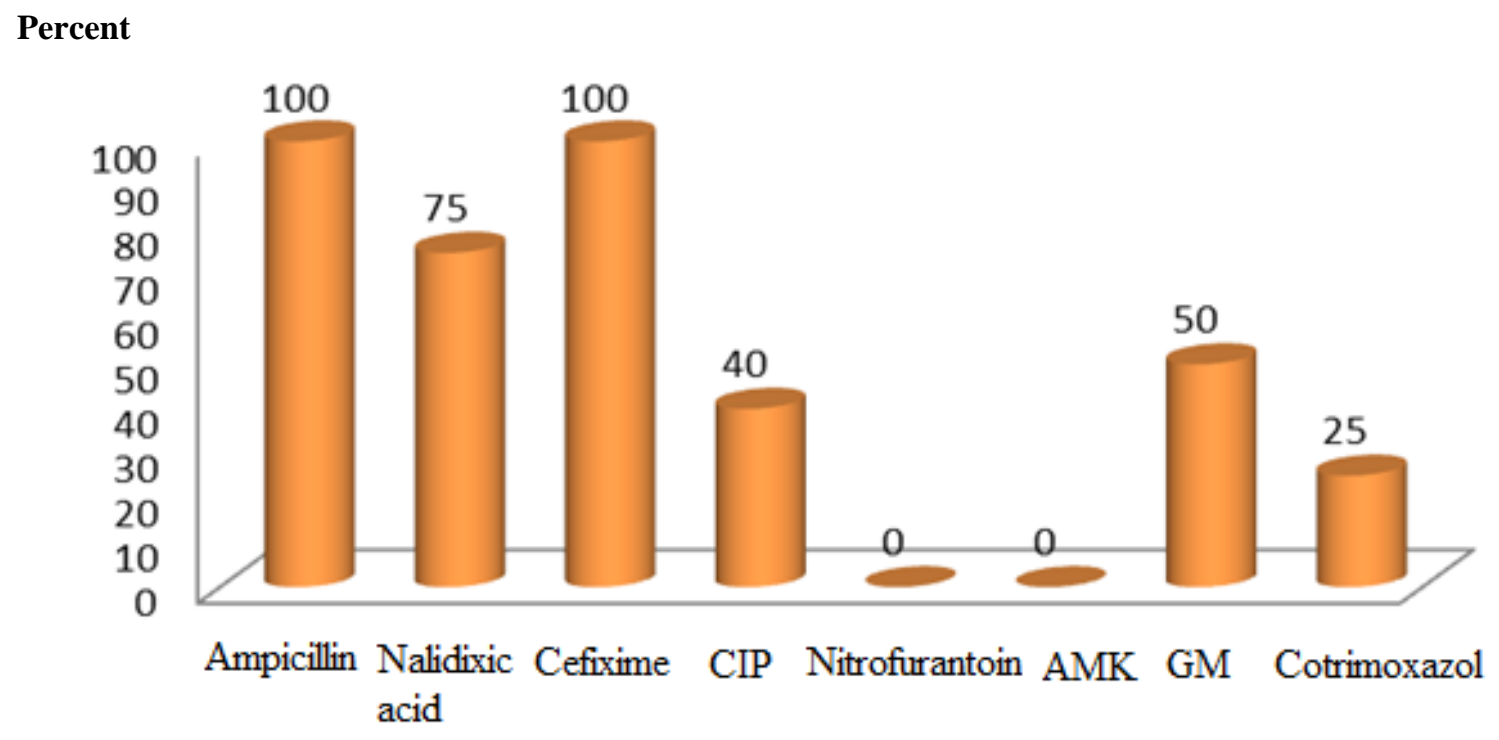

Antibiotic

Figure 3. Antibiotic resistance in Coagulase-positive Staphylococci

$\mathrm{CIP}=$ Ciprofloxacin, $\mathrm{AMK}=$ Amikacin, and GM= Gentamicin 


\section{Percent}

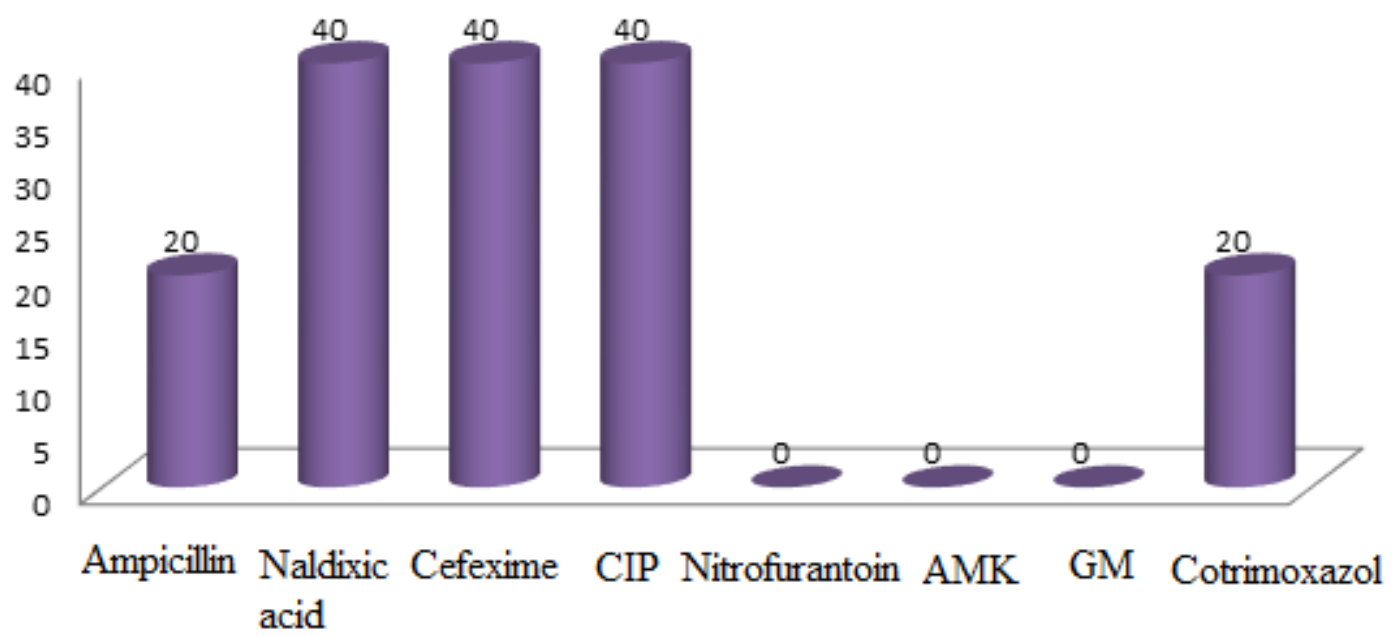

\section{Antibiotic}

Figure 4. Antibiotic resistance in Enterobacter

$\mathrm{CIP}=$ Ciprofloxacin, $\mathrm{AMK}=$ Amikacin, and $\mathrm{GM}=$ Gentamicin

\section{Percent}
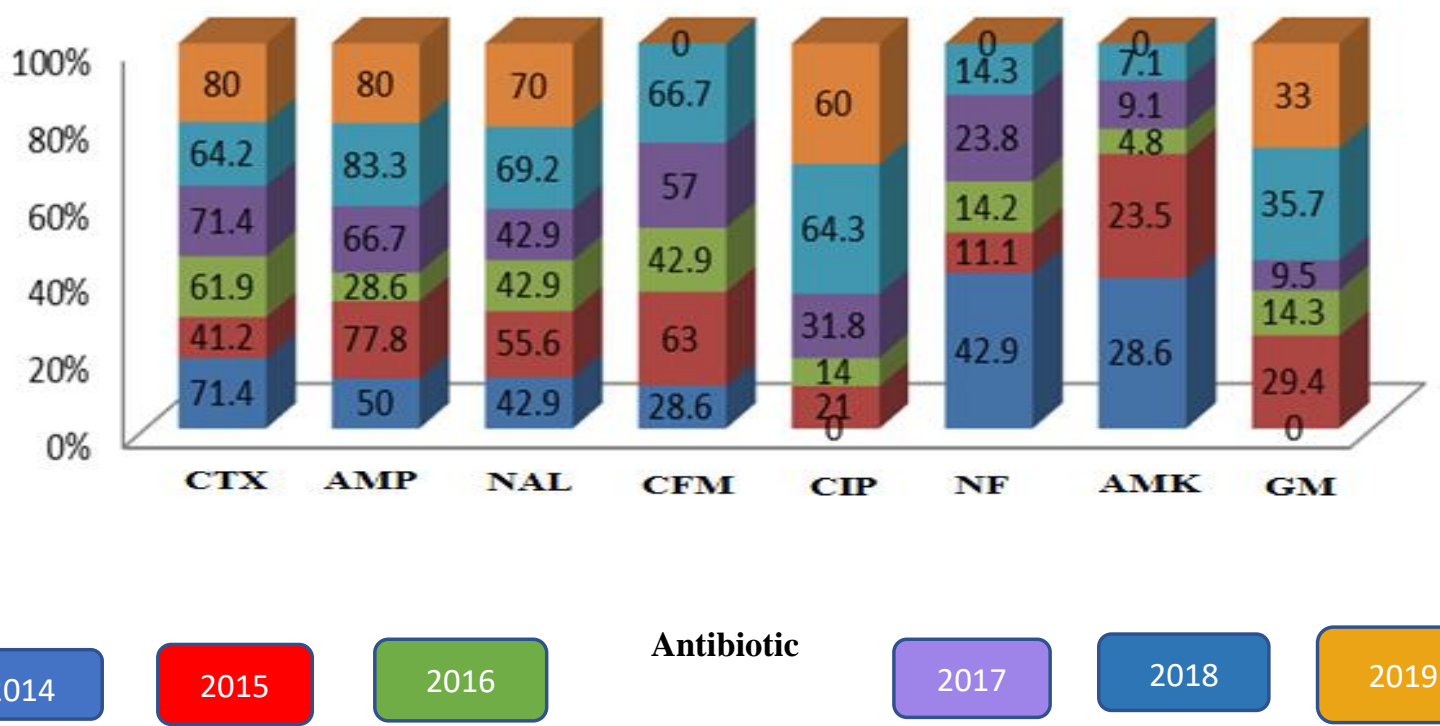

Antibiotic

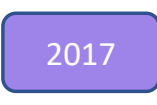

\section{8}

2019

Figure 5. Trend of antibiotic resistance during 2014 to 2019

$\mathrm{CIP}=$ Ciprofloxacin, $\mathrm{GM}=$ Gentamicin, $\mathrm{IPM}=$ Imipenem, $\mathrm{V}=$ Vancomycin AMK= Amikacin, $\mathrm{CFM}=$ Cefixime, $\mathrm{CTX}=$ Cotrimoxazole, $\mathrm{FEP}=$ Cefepime, $\mathrm{NAL}=$ Nalidixic acid, $\mathrm{AMP}=$ Ampicillin and $\mathrm{NF}=$ Nitrofurantoin 


\section{Urinary (kidney) stone}

Out of 101 patients with urinary tract infections, 55 had ultrasound of the urinary tract, of which only 5 were found to be urinary (kidney) stone. Four cases of urinary (kidney) stone were associated with $E$. coli and one case with Klebsiella. No significant relationship was found between the stone and the type of bacteria. In each of the years 2014 to 2017, one stone was observed each year and in 2018, two stones. There was also no significant relationship between stone and year. In people who had stones, the mean age was 29.6 and in people who did not have stones, the mean age was 37.3, but there was no significant relationship between stones and age.

\section{Antibiotic Resistance}

In this study, the highest resistance among different bacteria, on average, was related to cotrimoxazole $(62.5 \%)$, then ampicillin (61.4\%), nalidixic acid $(51.8 \%)$, cefixime $(50.8 \%)$, ciprofloxacin $(29.5 \%)$, gentamicin $(20.5 \%)$, nitrofurantoin $(17.6 \%)$, and amikacin $(11 \%)$ respectively. Vancomycin was tested on 19 isolates that $78.9 \%$ of the bacteria were resistant. Imipenem was also tested on 25 isolates that were only $4 \%$ resistant. Cefepime was also tested on 8 isolates, 3 isolates of which were resistant. E. coli has the highest resistance to cotrimoxazole and the lowest resistance to nitrofurantoin (figure 2). Also, vancomycin was tested on 9 isolates, of which $6(66.7 \%)$ were resistant to vancomycin. No isolate has been resistant to imipenem.

Staphylococcus coagulase positive had the highest resistance to cefixime and ampicillin. One isolate was resistant to imipenem and vancomycin and two isolates were resistant to cefoxitin (figure 3). Pseudomonas also had the highest resistance to nalidixic acid and cotrimoxazole. There was no resistance to vancomycin and imipenem. Klebsiella has the highest resistance to nitrofurantoin. Also, three isolates of Klebsiella in this study were resistant to vancomycin. But in Enterobacter, the highest resistance to ciprofloxacin, cefixime and nalidixic acid was observed (figure 4).

Three isolates in Enterobacter were resistant to vancomycin and one isolates was resistant to cefepime. Resistance to gentamicin, amikacin and nitrofurantoin was also not observed in this bacterium. Also, resistance in bacteria by year, cotrimoxazole has been high in all years and resistance to ampicillin has been high in all years except 2015. There has been a significant difference in resistance to ciprofloxacin, and in 2018 and 2019, resistance to this antibiotic increased. Also, Resistance to nalidixic acid increased in 2018 and 2019. Figure 5 shows the trend of antibiotic resistance by year with different colors.

\section{Discussion}

In most cases UTI are treated experimentally before the infectious agent and its pattern of antibiotic resistance are detected. Continuation of experimental administration in any region without knowledge of the common causes of infection and their resistance pattern increases the prevalence of antibiotic resistance. Therefore, to properly treat patients and choose the right antibiotics, it is necessary to determine the pattern of antibiogram and the prevalence of various bacteria in urinary tract infections. In current study,

E. coli was the most common bacterium, followed by Klebsiella spp. and Enterobacter and coagulasepositive Staphylococcus. In a similar study by Heidari et al., In Iran, E. coli was the most common bacterium isolated from patients with urinary tract infections, but after that, coagulase-positive Staphylococcus was the most abundant (12). Also, in a study Isberg et al., $E$. coli had the highest prevalence of urinary tract infections (13). A study in Nigeria found that E. coli and coagulase-positive Staphylococcus were most common in UTI of women, which is consistent with this study (14).

In this study, all isolates had the highest resistance to co-trimoxazole, Ampicillin and Nalidixic acid and had lowest resistance to imipenem. Saleh et al., Show that the sum of different isolates had the highest resistance to cotrimoxazole, which is consistent with our study. They also showed E. coli has the highest resistance to nalidixic acid (54.18\%), which differs from the results of the present study (15). In a similar study, Baghani et al., Showed the lowest resistance in imipenem (2). In a similar study, Isvand et al., Also showed that E. coli has the lowest resistance to nitrofurantoin (3). In a similar study conducted by Ghadage et al., Nitrofurantoin is a best antibiotic for the treatment of UTI and isolated bacteria have shown high resistance to ampicillin and cotrimoxazole.

In the present study, nitrofurantoin and amikacin are recommended to cure urinary tract infections (16). Also, in our study, the frequency of urinary tract stones was about $5 \%$. Four cases of kidney stones were associated with E. coli and one case with Klebsiella. The prevalence of urinary tract stones is increasing, the main reason for which is the change in people's lifestyle and urinary tract infection. In a similar study conducted by Mohkam et al., the prevalence of kidney stones was $17 \%$ and $19.8 \%$ of urinary tract stones were associated with urinary tract infections (17). Furthermore, Nito et al. Show that people who are 
familial are prone to stone making if encounter by $E$. coli, they are more likely to produce infectious stones (18).

\section{Conclusion}

The results of the present study showed that during this 6-year period, resistance to ciprofloxacin increased over time. Also, resistance to other antibiotics such as cotrimoxazole, ampicillin, nalidixic acid and cefixime is high and this could be an alarm signal for the Healthcare. Therefore, physicians' awareness of antibiotics profile in any country is very importance. Therefore, controlling the increase of this resistance is needed to prevent the over-the-counter use of antibiotics.

\section{References}

1. Tan, C.W., Chlebicki, M.P. Urinary tract infections in adults. Singapore Med J 2016; 57(9):48590.

2. Baghani, A.H., Ekrami, T.M., Haghighi, F., Tabarraie, Y. Common Bacterial Factors of Urinary Tract Infections and Determining their Antibiotic Resistance in Hospitalized and out Patients Referred to the Vase'ee Hospital in Sabzevar in 2016. J Sabzevar Uni Med Sci 2018; 25(5):687-693.

3. Isvand A, Yahyavi M, Asadi SM, Kooti W, Davoodi JZ. The study of bacteriological factors and antibiotic resistance in women with uti referring to the razi laboratory in Dezful. J Ilam Uni Med Sci 2014; 22(4):199-205.

4. Scales, C.D., Tasian, G.E., Schwaderer, A.L., Goldfarb, D.S., Star, R.A., Kirkali, Z. Urinary Stone Disease: Advancing Knowledge, Patient Care, and Population Health. Clin J Am Soc Nephrol 2016; 11(7):1305-1312.

5. Tanagho, E.A., McAninch, J.W., Smith S. General urology: Lange Medical Books,McGrawHill; 2004.

6. Parks, J.H., Coe, F.L. The financial effects of kidney stone prevention. Kidney Int 1996 ;50(5):170612.

7. Hulton, S. Evaluation of urinary tract calculi in children. Arch Dis childhood. 2001; 84(4):320-3.

8. Bichler, K.H., Eipper, E., Naber, K., Braun, V., Zimmermann, R., Lahme, S. Urinary infection stones. Int J Antimicrob Agents 2002; 19(6):488-98.

9. Yongzhi, L., Shi, Y., Jia, L., Yili, L., Xingwang, Z., Xue, G. Risk factors for urinary tract infection in patients with urolithiasis - primary report of a single center cohort. BMC Urol 2018; 18(1):45.

10. Kakian, F., Zamzad, B., Gholipour, A., Zamanzad, K. Determination of antibiotic resistance and minimum inhibitory concentration of meropenem and imipenem growth in Klebsiella strains isolated from urinary tract infection in Shahrekord educational hospitals. J Shahrekord Uni Med Sci 2019; 21(2):805.

11. Kakian, F., Abadi, M., Zamanzad, B., Najafi, H., Amiri, M., Gholipour, A. Frequency assessment of OXA-10 and PER $\beta$-lactamase genes and determination of minimum inhibitory concentration in klebsiella strains isolated from urinary tract infections. Jundishapur J Microb 2019; 12(2):e65500.

12. Heidari-soureshjani, E., Heidari, M., Doosti, A. Epidemiology of urinary tract infection and antibiotic resistance pattern of E. coli in patients referred to Imam Ali hospital in Farokhshahr, Chaharmahal va Bakhtiari, Iran. J Shahrekord Uni Med Sci 2013; 15(2):9-15.

13. Isberg, H.K., Melander, E., Hedin, K., Mölstad, S., Beckman, A. Uncomplicated urinary tract infections in Swedish primary care; etiology, resistance and treatment. BMC Infect Dis 2019;19(1):1-8.

14. Oli, A.N., Akabueze, V.B., Ezeudu, C.E., Eleje, G.U., Ejiofor, O.S., Ezebialu, I.U., Oguejiofor, C.B., Ekejindu, I.M., Emechebe, G.O., Okeke, K.N. Bacteriology and antibiogram of urinary tract infection among female patients in a tertiary health facility in south eastern Nigeria. Open Microb J 2017; 11:292-300.

15. Saleh, F., Soleiman Nejad, S., Bahrami Chegeni, F., Jafari, S., Javanmard, A., Rouhi, S., Shakib, P. Determination of Bacterial Factors causing Urinary Infections and its Antibiotic Resistance Patterns in Patients referred to Khorramabad Hospital, Iran. Pajouhan Scientific J 2018; 16(4):1-5.

16. Ghadage, D.P., Muley, V.A., Sharma, J., Bhore, A.V. Bacteriological profile and antibiogram of urinary tract infections at a tertiary care hospital. National J lab Med 2016; 5(4):20-4.

17. Mohkam, M., Mahani, F., Otoukesh, B., Sharifian, M., Dalirani, R., Hatamian, B. Epidemiology of urolithiasis among children hospitalized in Mofid hospital during 5 years. Pejouhandeh 2010; 15(3):133-136.

18. Nieto, V.G., Fernández, J.S., O’Hagan, M., Sancho, P.A., Yanes, M.I.L. A family history of renal lithiasis in children diagnosed of urinary tract infection by Escherichia coli. An Pediatr. 2018; 88(4):204-8. 